\title{
Effect of photodynamic therapy (PDT), posterior subtenon injection of triamcinolone acetonide with PDT, and intravitreal injection of ranibizumab with PDT for retinal angiomatous proliferation
}

\author{
This article was published in the following Dove Press journal: \\ Clinical Ophthalmology \\ 17 February 2012 \\ Number of times this article has been viewed
}

\author{
Saya Nakano' \\ Shigeru Honda' \\ Hideyasu $\mathrm{Oh}^{2}$ \\ Mihori Kita ${ }^{2}$ \\ Akira Negi \\ 'Department of Surgery, Division \\ of Ophthalmology, Kobe University \\ Graduate School of Medicine, Kobe, \\ ${ }^{2}$ Department of Ophthalmology, \\ Hyogo Prefectural Amagasaki \\ Hospital, Amagasaki, Japan
}

Background: The purpose of this work was to compare the efficacy of photodynamic therapy (PDT) with or without posterior subtenon injections of triamcinolone acetonide (STA) or intravitreal injections of ranibizumab (IVR) for retinal angiomatous proliferation (RAP).

Methods: Thirty-seven eyes from 33 consecutive patients with RAP were treated by PDT monotherapy (Group 1), PDT combined with STA (Group 2), or PDT combined with IVR (Group 3). The best-corrected visual acuity, greatest linear dimension, central retinal thickness, and number of treatments were compared among the three groups.

Results: The change in mean best-corrected visual acuity (logMAR) at month 3, 6, and 12 after the initial treatment was better in Group $2(-0.13,-0.23$, and -0.21 , respectively) and Group $3(-0.018,0.0028$, and -0.0067 , respectively) than in Group $1(0.13,0.19$, and 0.23 , respectively); Group 1 versus Group 2 was statistically significant $(P=0.018)$. The mean central retinal thickness was reduced from baseline in all groups, but the reduction amplitude was significantly greater in Group 2 than in Group 1 and Group 3. The mean number of treatments was significantly lower in Group $2(1.1 \pm 0.4)$ and Group $3(1.5 \pm 0.5)$ than in Group $1(2.9 \pm 0.9)$ in the 12 months after the initial treatment.

Conclusion: Treatment with STA + PDT may be an effective therapy for RAP lesions over 12 months of follow-up.

Keywords: retinal angiomatous proliferation, photodynamic therapy, triamcinolone acetonide, ranibizumab, combined therapy

\section{Introduction}

Age-related macular degeneration (AMD) is a leading cause of central vision loss in the elderly in industrialized countries. ${ }^{1}$ The number of patients with AMD has increased remarkably over the years, and a further increase in patients with severe visual impairment due to AMD is anticipated. ${ }^{2}$ Advanced AMD is clinically classified into atrophic AMD and exudative AMD. Exudative AMD is further classified into typical neovascular AMD, polypoidal choroidal vasculopathy, and retinal angiomatous proliferation (RAP). ${ }^{1}$ These phenotypes are known to have different characteristics in their natural courses and their responses to interventions, such as photodynamic therapy (PDT) and antivascular endothelial growth factor (VEGF) therapy, although the reasons are unknown. ${ }^{3-5}$ Several studies have reported a poor response to PDT monotherapy in patients with RAP lesions. ${ }^{6,7}$ Hence, it is common to perform PDT combined with intravitreal injections of triamcinolone acetonide (IVT) or anti-VEGF
Correspondence: Shigeru Honda Department of Surgery, Division of Ophthalmology, Kobe University Graduate School of Medicine, 7-5-2 Kusunoki-cho, Chuo-ku, Kobe 650-0017, Japan Tel +8I 783826048

Fax +8I 783826059

Email sighonda@med.kobe-u.ac.jp 
therapy for RAP. ${ }^{8,9}$ Several studies were conducted to address the comparative effectiveness between PDT monotherapy and the combination of PDT with IVT or anti-VEGF therapy against RAP lesions, but their conclusions were not consistent. ${ }^{10,11}$ Rouvas et al reported that IVT + PDT was more effective than intravitreal ranibizumab (IVR) + PDT for RAP lesions. ${ }^{10}$ In contrast, Saito et al reported that intravitreal bevacizumab + PDT was likely to be more effective than IVT + PDT in a Japanese RAP cohort. ${ }^{11}$ This discrepancy might reflect the different anti-VEGF agents used in the two studies, so more replication studies are needed.

A posterior subtenon injection of triamcinolone acetonide (STA) is an alternative method to deliver triamcinolone acetonide to the posterior retina. Although IVT may cause an elevation in intraocular pressure and cataracts as complications, ${ }^{12,13}$ STA might have fewer side effects in terms of inducing intraocular pressure elevation or cataracts than IVT because STA should act on the retina transsclerally and thus affect the lens and trabecular meshwork less than IVT. ${ }^{14,15}$ However, to our knowledge, only a few published studies have compared the effectiveness of PDT combined with STA. ${ }^{13}$ Therefore, in this study, we performed a comparative assessment between STA + PDT, IVR + PDT, and PDT alone in RAP patients.

\section{Subjects and methods}

This study was approved by the institutional review board of the Kobe University Graduate School of Medicine, and was conducted in accordance with the Declaration of Helsinki. Written informed consent was obtained from all subjects. All cases in this study were Japanese individuals recruited from the Department of Ophthalmology at Kobe University Hospital and Hyogo Prefectural Amagasaki Hospital in Japan.

This was a retrospective study of 37 eyes from 33 consecutive patients with RAP treated and followed up for more than 6 months. All patients received detailed ophthalmic examinations, including best-corrected visual acuity (BCVA) measurements, slit lamp biomicroscopy of their fundi, color fundus photography, fluorescein angiography, indocyanine green angiography, and optical coherence tomography. Visual acuities were determined using a Landolt $\mathrm{C}$ chart, and were converted to a logarithm of the minimum angle of resolution ( $\log$ MAR) values for calculation. The diagnosis and staging of RAP was performed as previously described. ${ }^{16}$ Patients with past histories of retinal vessel occlusion, uveitis, rhegmatogenous retinal detachment, or glaucoma were excluded.

Fourteen eyes from 12 consecutive patients recruited by May 2006 were treated by PDT monotherapy (Group 1), 12 eyes from 10 consecutive patients recruited from June 2006 to March
2009 were treated by PDT combined with STA (Group 2), and 11 eyes from 11 consecutive patients recruited thereafter were treated by PDT combined with IVR (Group 3). No patients in this study received previous therapy except for one patient in Group 2 who underwent PDT monotherapy 4 months earlier. For STA + PDT, a small incision was made in the lower temporal conjunctiva, and $20 \mathrm{mg}$ of triamcinolone acetonide was injected retrobulbarly with a blunt needle 4-7 days before PDT. For IVR + PDT, $0.3 \mathrm{mg}$ of ranibizumab was injected intravitreally with a 30-gauge needle 3-7 days before PDT. Patients were examined 3, 6, 9, and 12 months after the initial treatment, and were retreated if persistence or recurrence of intraretinal, subretinal, or subretinal pigment epithelium fluid, or any increase in retinal thickness was found by funduscopy or optical coherence tomography. The retreatment was done according to the same protocol as for the initial treatment.

For statistical analysis, we compared the gender, age, BCVA, greatest linear dimension, and central retinal thickness at baseline among the three groups. Changes in BCVA and central retinal thickness were then compared every 3 months until month 12 after the initial treatment. The number of treatments performed during the first 12 months after the initial treatment was compared among the groups. To evaluate the influence of STA on intraocular pressure, intraocular pressure values before and 2 weeks after STA were measured for the STA + PDT group. The Wilcoxon signed-rank test was performed to compare any two time points within the group and an analysis of variance was used to make a comparison between the groups. $P$ values of 0.05 or less were considered to be statistically significant. StatView-J software (v 5.0; Abacus Corporation, Baltimore, MD) was used for statistical analyses.

\section{Results}

A summary of the data for the RAP patients is shown in Table 1. No differences were detected in baseline parameters between the three groups. The transition of values in mean BCVA $(\log \mathrm{MAR})$ and mean central retinal thickness are shown in Table 2. The change in mean BCVA at months 3, 6, and 12 after initial treatment was better in Group $2(-0.13,-0.23$, and -0.21$)$ and Group $3(-0.018,0.0028$, and -0.0067$)$ than in Group $1(0.13,0.19$, and 0.23$)$; Group 1 versus Group 2 was statistically significant $(P=0.018$, Figure 1$)$. The mean BCVA was significantly better than baseline in Group 2 at 6 and 12 months after the initial treatment $(P=0.012$ and 0.025 , respectively). In contrast, the mean BCVA in Group 3 had deteriorated by 12 months after the initial treatment, although it was not significant $(P=0.12)$. The mean central retinal thickness was reduced from baseline after initial treatment in 
Table I Baseline parameters of participants

\begin{tabular}{|c|c|c|c|c|}
\hline & $\begin{array}{l}\text { Group I } \\
(n=14)\end{array}$ & $\begin{array}{l}\text { Group } 2 \\
(n=12)\end{array}$ & $\begin{array}{l}\text { Group } 3 \\
(n=I I)\end{array}$ & $P$ value \\
\hline Gender (male/female) & $11 / 1$ & $6 / 4$ & $4 / 7$ & $0.022^{\dagger}$ \\
\hline Age (mean $\pm S D$, years) & $82.3 \pm 4.1$ & $78.2 \pm 5.7$ & $80.3 \pm 7.2$ & $0.10^{*}$ \\
\hline RAP stage I & 1 & 3 & 3 & $0.6 \mathrm{I}^{\dagger}$ \\
\hline Stage 2 & 6 & 4 & 5 & \\
\hline Stage 3 & 7 & 5 & 3 & \\
\hline GLD (mean \pm SD) & $4737 \pm 1704$ & $3948 \pm 1238$ & $3291 \pm 1418$ & $0.10 *$ \\
\hline Baseline BCVA logMAR (mean \pm SD) & $0.82 \pm 0.47$ & $0.78 \pm 0.55$ & $0.84 \pm 0.37$ & $0.84 *$ \\
\hline
\end{tabular}

Notes: Group I, PDT monotherapy; Group 2, STA + PDT; Group 3, IVR + PDT; *Kruskal-Wallis test; ${ }^{\dagger}$ Chi-square test.

Abbreviations: GLD, greatest linear dimension; BCVA, best-corrected visual acuity; PDT, photodynamic therapy; STA, subtenon injection of triamcinolone acetonide; IVR, intravitreal injection of ranibizumab; SD, standard deviation.

all groups and the decrease was significant by 12 months in the $\mathrm{STA}+\mathrm{PDT}$ and IVR + PDT groups (Figure 2). The reduction amplitude was significantly greater in Group 2 than in Group 1 and Group $3(P=0.024$ and $P=0.033$, respectively, for Group 1 versus Group 2 and Group 2 versus Group 3). In the cases followed up for more than 12 months after initial treatment, the mean number of treatments was significantly lower in Group $2(1.1 \pm 0.4, \mathrm{n}=12)$ and Group $3(1.5 \pm 0.5$, $\mathrm{n}=9)$ than in Group $1(2.9 \pm 0.9, \mathrm{n}=14)$ over 12 months after initial treatment $(P<0.0001, P=0.0004$, and $P=0.15$ for Group 1 versus Group 2, Group 1 versus Group 3 and Group 2 versus Group 3, respectively, Mann-Whitney test) (Figure 3). In Group 2, the mean intraocular pressure before and after STA was $13.8 \pm 3.4 \mathrm{mmHg}$ and $16.8 \pm 6.1 \mathrm{mmHg}$, respectively, ( $P=0.18$, two-tailed paired $t$-test). No ocular or systemic complications were found or self-reported in the present cases.

\section{Discussion}

We compared the effects of PDT monotherapy, STA + PDT, and IVR + PDT in patients with RAP lesions, and found that

Table 2 Transition in best-corrected visual acuity and central retinal thickness of each group

\begin{tabular}{llll}
\hline & Group I & Group 2 & Group 3 \\
\hline $\begin{array}{lll}\text { BCVA (logMAR) } \\
\text { Baseline }\end{array}$ & $0.82 \pm 0.47$ & $0.78 \pm 0.55$ & $0.82 \pm 0.38$ \\
3 months & $0.95 \pm 0.36$ & $0.66 \pm 0.37$ & $0.80 \pm 0.44$ \\
6 months & $1.01 \pm 0.3 \mathrm{I}$ & $0.56 \pm 0.37^{*}$ & $0.82 \pm 0.40$ \\
I2 months & $1.05 \pm 0.32$ & $0.57 \pm 0.35^{*}$ & $0.8 \mathrm{I} \pm 0.42$ \\
CRT ( $\mu$ m) & & & \\
Baseline & $315 \pm 93$ & $358 \pm 88$ & $314 \pm 102$ \\
3 months & $268 \pm 104$ & $204 \pm 72^{*}$ & $21 \mathrm{I} \pm 5 \mathrm{I}$ \\
6 months & $260 \pm 93$ & $202 \pm 84^{*}$ & $223 \pm 5 \mathrm{I}^{*}$ \\
I2 months & $263 \pm 84$ & $24 \mathrm{I} \pm 74^{*}$ & $202 \pm 46^{*}$ \\
\hline
\end{tabular}

Notes: Group I, PDT monotherapy; Group 2, STA + PDT; Group 3, IVR + PDT; $* P<0.05$ versus baseline.

Abbreviations: CRT, central retinal thickness; BCVA, best corrected visual acuity; PDT, photodynamic therapy; STA, subtenon injection of triamcinolone acetonide; IVR, intravitreal injection of ranibizumab. the visual outcome was significantly better in those patients who underwent STA + PDT than in those treated with PDT monotherapy, although STA + PDT and IVR + PDT showed no significant difference in visual outcome. The mean number of treatments required per year was significantly lower in the STA + PDT and IVR + PDT groups than for the PDT monotherapy group. In addition, STA + PDT did not cause a significant elevation of intraocular pressure.

Currently, RAP is thought to be the most difficult subtype of exudative AMD to treat. ${ }^{7}$ Because previous studies have demonstrated an insufficient effect of PDT monotherapy for RAP lesions, ${ }^{6,7}$ most of the recent studies have focused on the effectiveness of PDT combined with IVT or antiVEGF therapy. ${ }^{8-11}$ However, few reports have evaluated the effects of STA + PDT for RAP. Montero et al reported that no better outcomes were observed in RAP patients treated with STA + PDT than in those treated with PDT monotherapy. ${ }^{13}$ They administered $40 \mathrm{mg}$ of triamcinolone acetonide immediately after PDT, which resulted in no significant difference in outcomes as compared with PDT monotherapy. However, we used $20 \mathrm{mg}$ of triamcinolone acetonide 4-7 days before PDT, and obtained significantly better outcomes as compared with PDT monotherapy in RAP patients. This difference might be due to the insufficient time for transscleral diffusion of triamcinolone acetonide when it was applied after PDT. ${ }^{14,15}$ Rouvas et al reported favorable outcomes for IVT + PDT when IVT was performed $7 \pm 3$ days before PDT. ${ }^{10}$ The mechanism by which triamcinolone acetonide works to improve the outcome of PDT is still a matter of speculation. An inflammatory response and upregulation of VEGF have been reported after application of PDT. ${ }^{17,18}$ Because triamcinolone acetonide has antiangiogenic, anti-inflammatory, and anti-VEGF effects, ${ }^{19,20}$ the combination of PDT and triamcinolone acetonide may reduce the inflammatory response and upregulation of VEGF associated with choroidal neovascularization and PDT. 


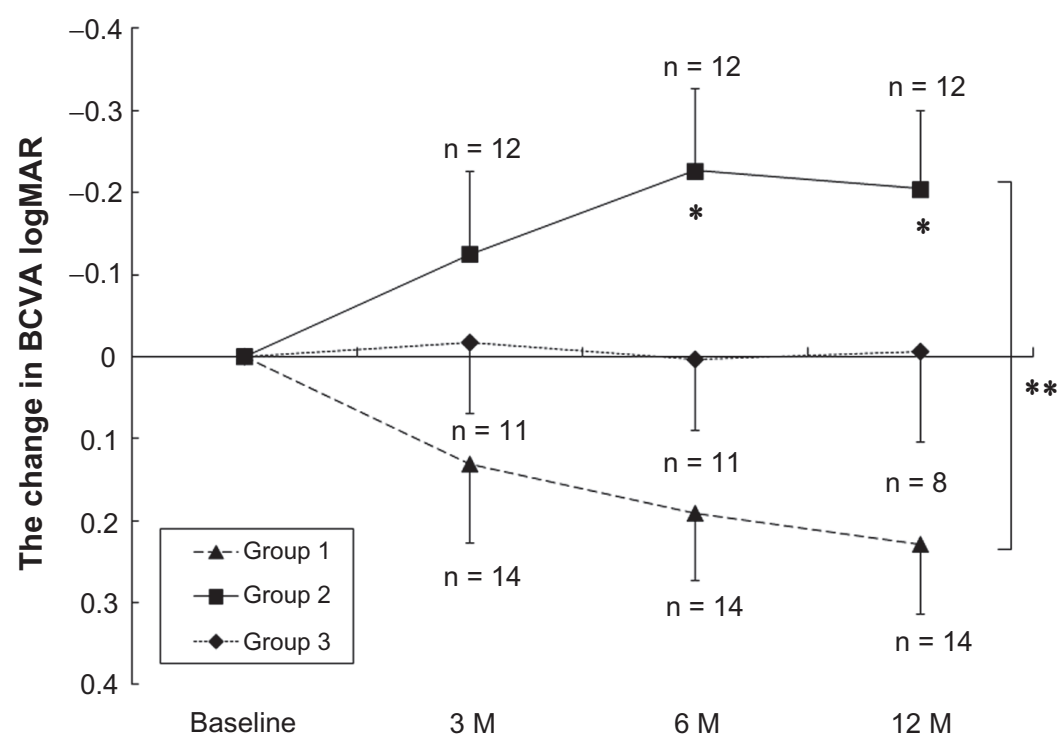

Figure I Changes in the BCVA of RAP patients after PDT, STA + PDT, and IVR + PDT. The BCVA was determined using the Landolt C chart, and is presented as decimal visual acuities. Triangles with dashed line: PDT (Group I); squares with solid line: STA + PDT (Group 2); diamonds with dot line: IVR + PDT (Group 3).

Notes: Values represent mean \pm SEM; $* P<0.05$ compared to baseline; **P $<0.05$ between Group I and Group 2.

Abbreviations: BCVA, best corrected visual acuity; IVR, intravitreal injections of ranibizumab; RAP, retinal angiomatous proliferation; PDT, photodynamic therapy, STA, subtenon injections of triamcinolone acetonide.

The significantly greater central retinal thickness reduction after STA + PDT than after IVR + PDT in the present study might reflect a difference in anti-inflammatory and antiVEGF effects between STA and IVR, which was possibly associated with the better outcome, although not significant, in the post-treatment BCVA for the STA + PDT group than for the IVR + PDT group. Although we performed IVR 3-7 days before PDT, the timing of IVR might be too early because Debefe et al reported that ranibizumab should be administered within 24 hours after PDT in accordance with their experimental results. ${ }^{21} \mathrm{~A}$ recent report showed favorable visual outcomes after IVR + PDT when PDT was performed 1-2 days after IVR. ${ }^{22}$ In addition, unlike previous reports, we did not add two extra monthly IVR after PDT in Group 3 to save treatment costs, which might reduce the effect of IVR + PDT. However, the change in logMAR BCVA in Group 3 was almost equivalent to that of the previous report ( 0.02 between baseline and at least 6 months after the first therapy) performing three IVR + one PDT as an initial treatment. ${ }^{10}$ There is another possibility that the effects of

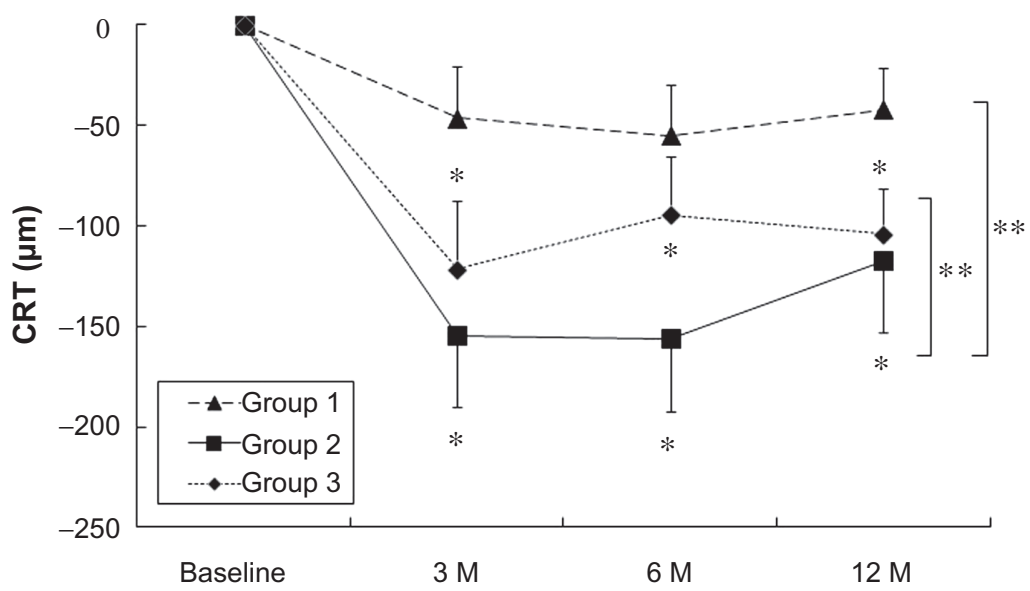

Figure 2 Changes in the CRT of RAP patients after PDT, STA + PDT, and IVR + PDT. Triangles with dashed line: PDT (Group I); squares with solid line: STA + PDT (Group 2); diamonds with dot line: IVR + PDT (Group 3).

Notes: Values represent mean \pm SEM. $* P<0.05$. **P $<0.05$ between Group I and Group 2, or between Group 2 and Group 3.

Abbreviations: CRT, central retinal thickness; IVR, intravitreal injections of ranibizumab; RAP, retinal angiomatous proliferation; PDT, photodynamic therapy, STA, subtenon injections of triamcinolone acetonide. 


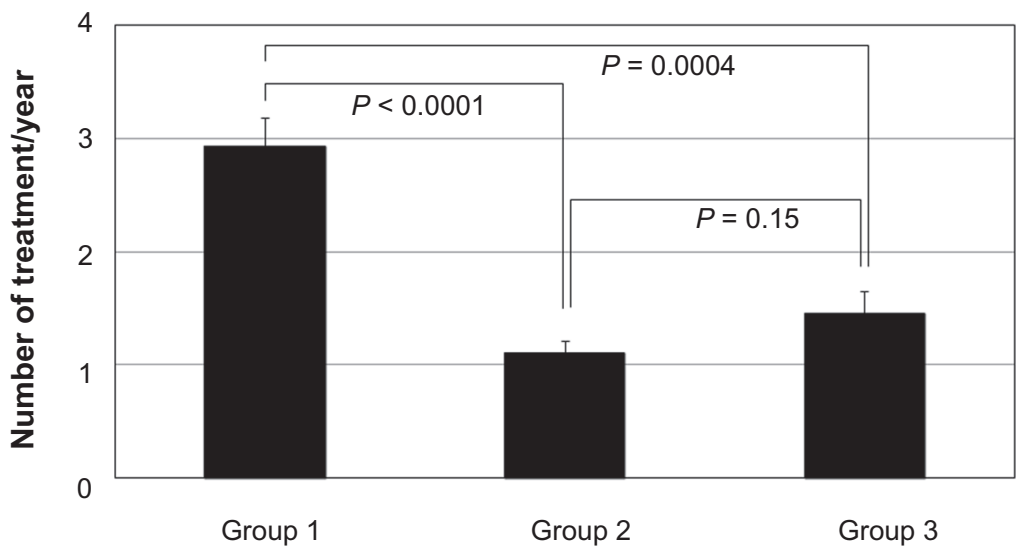

Figure 3 Number of treatments performed in 12 months. Note: Values represent mean + SEM.

STA remained for several months after PDT and inhibited regrowth of neovascular tracts, reducing the number of treatments required to suppress the RAP lesions, and thus resulted in reduced cumulative retinal damage caused by PDT. Because a single STA is thought to have antiangiogenic and anti-inflammatory effects lasting up to 3 months $^{23}$ while a single dose of IVR can work for a month, STA + PDT might have an advantage to suppress the RAP lesion longer than IVR + PDT. Conversely, IVR + PDT may necessitate some additional IVR during follow-up period.

Our results showed that the best mean BCVA was obtained at 6 months after the initial STA + PDT, but this was reduced by 12 months, mainly due to reactivation of RAP lesions in some cases. It is interesting that a reactivation of RAP lesions at 6 months after treatment was previously reported in cases treated with IVT + PDT. ${ }^{24} \mathrm{~A}$ previous review article mentioned that the best improvement in BCVA was achieved at 6 months after initial PDT + IVT, and that the effects faded by 12 months, with a high incidence of cataracts. ${ }^{9}$ However, in our study, no patient in the STA + PDT group showed any progression of cataracts during the follow-up period. In addition, the incidence of intraocular pressure elevation was reported less often with STA than with IVT. ${ }^{25}$

Currently, many treatment procedures are being tested and compared to establish the best strategy for treating RAP lesions. Among them, anti-VEGF therapy is the most investigated modality which could be applied alone $e^{26,27}$ or combined with PDT. ${ }^{10,11,28}$ However, IVR + PDT is likely to be a very expensive therapy and intravitreal bevacizumab + PDT is not possible without off-label use of bevacizumab under current circumstances. If STA + PDT showed similar or better outcomes to PDT+ anti-VEGF therapy or anti-VEGF monotherapy, there is a greater cost-effectiveness for patients. In fact, the improvement in BCVA $(-0.22 \pm 0.34 \log \mathrm{MAR}$ units) with STA + PDT in this study was almost equivalent to the average of previous reports $(-0.17 \pm 0.12 \log$ MAR units) with anti-VEGF monotherapy. ${ }^{9,29}$

The major limitations of the present study were its nonrandomized and retrospective nature and the small number of subjects. Hence, it is important to evaluate the results of a randomized controlled trial for STA + PDT with a larger number of subjects to determine the efficacy of this therapy, particularly against RAP. Therefore, further investigation will be needed to determine the correct interventions for RAP.

In conclusion, STA + PDT may be an effective therapy for RAP lesions during the first 12 months after treatment, although the effects need to be further evaluated.

\section{Acknowledgments}

This study was supported by a grant-in aid (23592567) from the Ministry of Education, Science, and Culture, Tokyo, Japan (SH), and by a grant from the Takeda Science Foundation (SH).

\section{Disclosure}

No authors have any financial or conflicting interests to disclose in this work.

\section{References}

1. Cook HL, Patel PJ, Tufail A. Age-related macular degeneration: diagnosis and management. Br Med Bull. 2008;85:127-149.

2. Rein DB, Wittenborn JS, Zhang X, et al. Forecasting age-related macular degeneration through the year 2050: the potential impact of new treatments. Arch Ophthalmol. 2009;127:533-540.

3. Honda S, Kurimoto Y, Kagotani Y, et al. Photodynamic therapy for typical age-related macular degeneration and polypoidal choroidal vasculopathy: a 30-month multicenter study in Hyogo, Japan. Jpn J Ophthalmol. 2009;53:593-597.

4. Honda S, Imai H, Yamashiro K, et al. Comparative assessment of photodynamic therapy for typical age-related macular degeneration and polypoidal choroidal vasculopathy: a multicenter study in Hyogo prefecture, Japan. Ophthalmologica. 2009;223:333-338. 
5. Lim JY, Lee SY, Kim JG, Lee JY, Chung H, Yoon YH. Intravitreal bevacizumab alone versus in combination with photodynamic therapy for the treatment of neovascular maculopathy in patients aged 50 years or older: 1-year results of a prospective clinical study. Acta Ophthalmol. 2010. [Epub ahead of print.]

6. Boscia F, Furino C, Sborgia L, Reibaldi M, Sborgia C. Photodynamic therapy for retinal angiomatous proliferations and pigment epithelium detachment. Am J Ophthalmol. 2004;138: 1077-1079.

7. Boscia F, Parodi MB, Furino C, Reibaldi M, Sborgia C. Photodynamic therapy with verteporfin for retinal angiomatous proliferation. Graefes Arch Clin Exp Ophthalmol. 2006;244:1224-1232.

8. Krebs I, Krepler K, Stolba U, Goll A, Binder S. Retinal angiomatous proliferation: combined therapy of intravitreal triamcinolone acetonide and PDT versus PDT alone. Graefes Arch Clin Exp Ophthalmol. 2008;246:237-243.

9. Gupta B, Jyothi S, Sivaprasad S. Current treatment options for retinal angiomatous proliferans (RAP). Br J Ophthalmol. 2010;94:672-677.

10. Rouvas AA, Papakostas TD, Vavvas D, et al. Intravitreal ranibizumab, intravitreal ranibizumab with PDT, and intravitreal triamcinolone with PDT for the treatment of retinal angiomatous proliferation: a prospective study. Retina. 2009;29:536-544.

11. Saito M, Shiragami C, Shiraga F, Kano M, Iida T. Comparison of intravitreal triamcinolone acetonide with photodynamic therapy and intravitreal bevacizumab with photodynamic therapy for retinal angiomatous proliferation. Am J Ophthalmol. 2010;149: $472-481$.

12. Ruiz-Moreno JM, Montero JA, Barile S, Zarbin MA. Photodynamic therapy and high-dose intravitreal triamcinolone to treat exudative age-related macular degeneration: 1-year outcome. Retina. 2006;26:602-612.

13. Montero JA, Ruiz-Moreno JM, Sanabria MR, Fernandez-Munoz M. Efficacy of intravitreal and periocular triamcinolone associated with photodynamic therapy for treatment of retinal angiomatous proliferation. Br J Ophthalmol. 2009;93:166-170.

14. Mora P, Eperon S, Felt-Baeyens O, et al. Trans-scleral diffusion of triamcinolone acetonide. Curr Eye Res. 2005;30:355-361.

15. Lee SJ, Kim ES, Geroski DH, McCarey BE, Edelhauser HF. Pharmacokinetics of intraocular drug delivery of Oregon green 488-labeled triamcinolone by subtenon injection using ocular fluorophotometry in rabbit eyes. Invest Ophthalmol Vis Sci. 2008;49: 4506-4514.

16. Yannuzzi LA, Negrao S, Iida T, et al. Retinal angiomatous proliferation in age-related macular degeneration. Retina. 2001;21:416-434.
17. Tatar O, Adam A, Shinoda K, et al. Influence of verteporfin photodynamic therapy on inflammation in human choroidal neovascular membranes secondary to age-related macular degeneration. Retina. 2007;27:713-723.

18. Tatar O, Adam A, Shinoda K, et al. Expression of VEGF and PEDF in choroidal neovascular membranes following verteporfin photodynamic therapy. Am J Ophthalmol. 2006;142:95-104.

19. Penfold PL, Wen L, Madigan MC, King NJ, Provis JM. Modulation of permeability and adhesion molecule expression by human choroidal endothelial cells. Invest Ophthalmol Vis Sci. 2002;43:3125-3130.

20. Hori Y, Hu DE, Yasui K, Smither RL, Gresham GA, Fan TP. Differential effects of angiostatic steroids and dexamethasone on angiogenesis and cytokine levels in rat sponge implants. Br J Pharmacol. 1996;118:1584-1591.

21. Debefve E, Pegaz B, Ballini JP, van den Bergh H. Combination therapy using verteporfin and ranibizumab; optimizing the timing in the CAM model. Photochem Photobiol. 2009;85:1400-1408.

22. Saito M, Iida T, Kano M. Combined intravitreal ranibizumab and photodynamic therapy for retinal angiomatous proliferation. Am J Ophthalmol. 2011. [Epub ahead of print].

23. Shen L, You Y, Sun S, Chen Y, Qu J, Cheng L. Intraocular and systemic pharmacokinetics of triamcinolone acetonide after a single 40-mg posterior subtenon application. Ophthalmology. 2010;117:2365-2371.

24. Reche-Frutos J, Calvo-Gonzalez C, Donate-Lopez J, et al. Retinal angiomatous proliferation reactivation 6 months after high-dose intravitreal acetonide triamcinolone and photodynamic therapy. Eur J Ophthalmol. 2007;17:979-982.

25. Hirano Y, Ito T, Nozaki M, et al. Intraocular pressure elevation following triamcinolone acetonide administration as related to administration routes. Jpn J Ophthalmol. 2009;53:519-522.

26. Konstantinidis L, Mameletzi E, Mantel I, Pournaras JA, Zografos L, Ambresin A. Intravitreal ranibizumab (Lucentis) in the treatment of retinal angiomatous proliferation (RAP). Graefes Arch Clin Exp Ophthalmol. 2009;247:1165-1171.

27. Hemeida TS, Keane PA, Dustin L, Sadda SR, Fawzi AA. Longterm visual and anatomical outcomes following anti-VEGF monotherapy for retinal angiomatous proliferation. $\mathrm{Br} J$ Ophthalmol. 2010;94:701-705

28. Viola F, Mapelli C, Villani E, et al. Sequential combined treatment with intravitreal bevacizumab and photodynamic therapy for retinal angiomatous proliferation. Eye (Lond). 2010;24:1344-1351.

29. Parodi MB, Iacono P, Menchini F, et al. Intravitreal bevacizumab versus ranibizumab for the treatment of retinal angiomatous proliferation. Acta Ophthalmol. 2011. [Epub ahead of print].
Clinical Ophthalmology

\section{Publish your work in this journal}

Clinical Ophthalmology is an international, peer-reviewed journal covering all subspecialties within ophthalmology. Key topics include: Optometry; Visual science; Pharmacology and drug therapy in eye diseases; Basic Sciences; Primary and Secondary eye care; Patient Safety and Quality of Care Improvements. This journal is indexed on Submit your manuscript here: http://www.dovepress.com/clinical-ophthalmology-journal

\section{Dovepress}

PubMed Central and CAS, and is the official journal of The Society of Clinical Ophthalmology (SCO). The manuscript management system is completely online and includes a very quick and fair peer-review system, which is all easy to use. Visit http://www.dovepress.com/ testimonials.php to read real quotes from published authors. 